\title{
Multicentric Castleman's disease in HIV patients: a single-center cohort diagnosed from 2008 to 2018
}

\author{
Smaranda Gliga ${ }^{1} \cdot$ Hans Martin Orth ${ }^{1} \cdot$ Nadine Lübke $^{2} \cdot$ Jörg Timm $^{2} \cdot$ Tom Luedde $^{1} \cdot$ Björn-Erik Ole Jensen ${ }^{1}$
}

Received: 13 January 2021 / Accepted: 23 April 2021 / Published online: 4 May 2021

○) Springer-Verlag GmbH Germany, part of Springer Nature 2021, corrected publication 2021

\begin{abstract}
Purpose Castleman's disease (CD) is a well-established entity but there is a lack of available data regarding the management and therapy of HIV- and HHV-8-positive multicentric CD (MCD). We provide our own single-center experience with HIV-associated MCD.

Methods We performed a retrospective, descriptive study on a cohort of patients with MCD, diagnosed and admitted to the infectious diseases or intensive care unit in the University Hospital Düsseldorf between 2008 and 2018. Included patients had a previous or new HIV diagnosis and clinical signs resembling MCD with evidence of HHV-8 replication or histological diagnosis for MCD.

Results Nine male patients were included in the study. All patients were treated with Rituximab after diagnosis of MCD, with six of them acquiring resolution of symptoms. Three patients received tocilizumab additionally. Other treatment options included: splenectomy (2/9), valganciclovir (2/9), vincristine and siltuximab (1/9), ruxolitinib and Cytosorb ${ }^{\circledR}(2 / 9)$. The relapse rate was $44 \%$ (4/9) and the survival rate $87.5 \%$ after 1 year (8/9) and $71.4 \%$ after 3 years (5/7).

Conclusion The most effective first-line therapy and retreatment option remains rituximab. The effectiveness of other treatment options like splenectomy or different immunotherapeutic approaches requires confirmation in larger-scale studies.
\end{abstract}

Keywords Castleman's disease $\cdot \mathrm{HHV}-8 \cdot \mathrm{HIV} \cdot \mathrm{IL}-6 \cdot$ Rituximab $\cdot$ Ruxolitinib

\section{Introduction}

First described in the 1950s [1], Castleman's disease (CD) refers nowadays to a group of different entities of lymphoproliferative disorders. Diagnosis is usually confirmed histologically from entire enucleated lymph nodes. Three patterns have been described: the hyaline vascular type, the plasma cell type and more recently the plasmablastic multifocal CD. The extent of lymphadenopathy divides the disease into unicentric (localized) (UCD) and multicentric Castleman's disease (MCD). The hyaline vascular variant is mostly encountered in UCD

Smaranda Gliga and Hans Martin Orth contributed equally.

Smaranda Gliga

smaranda.gliga@med.uni-duesseldorf.de

1 Department of Gastroenterology, Hepatology and Infectious Diseases, University Hospital Düsseldorf, Heinrich Heine University, Düsseldorf, Germany

2 Institute of Virology, Heinrich Heine-University, University Hospital Düsseldorf, Düsseldorf, Germany patients, while the plasma cell variant is common in MCD patients and generally occurs in HIV-infected patients. This type usually involves generalized lymphadenopathy, splenomegaly and often shows symptoms resembling hyperinflammatory diseases or sepsis [2]. The third type develops into an aggressive disease and was initially described in the context of POEMS (polyneuropathy, organomegaly, endocrinopathy, monoclonal gammopathy and skin changes) syndrome [3].

Almost all HIV-associated cases of MCD and about $50 \%$ of HIV-negative MCD cases are associated with human herpes virus 8 (HHV-8), which is also involved in the pathogenesis of Kaposi's sarcoma (KS) and, therefore, sometimes referred to as KS-associated herpes virus (KSHV) [4]. This explains the frequent comorbidity of Kaposi's sarcoma and MCD, which has been described in $72 \%$ of HIV-1-infected CD patients [5, 6]. In addition to other clinical parameters, the HHV-8 DNA plasma level has been reported to be helpful in differentiating between Kaposi's sarcoma and MCD, although a reliable distinction could not be shown in another study $[4,7]$.

Interleukin-6 (IL-6) signaling plays a key role in the development of MCD. Monoclonal antibodies against IL-6 
receptors like tocilizumab and siltuximab showed promising results in the treatment of CD [8]. However, studies were only conducted in HIV-negative and predominantly also HHV-8-negative patients [8, 9]. Siltuximab is a reliable treatment option for newly diagnosed or previously treated "idiopathic" CD (HIV and HHV-8 negative) [9, 10]. Anecdotal cases of tocilizumab therapy in HIV- and HHV-8-positive patients have shown that this monoclonal antibody could be used as a first-line therapy but was unsuccessful for patients who relapsed during continuous treatment. A switch to Rituximab therapy often led to remission in these patients [11].

Rituximab, a monoclonal CD20 antibody targeting B cells is a successful first-line therapy in HIV-associated MCD [12]. A retrospective analysis of 52 patients with HIVassociated MCD showed a higher percentage of sustained remission and survival in patients receiving rituximabbased therapies in comparison to cytostatic therapy alone [13]. A disadvantage of Rituximab usage seems to be an exacerbation of KS in patients with previously diagnosed $\mathrm{KS}$ at treatment initiation, despite concomitant combination antiretroviral therapy (cART) [12]. However, treatment failure in HIV-positive patients with aggressive MCD and organ failure required additional chemotherapy $[14,15]$. The most commonly used regimens are similar to those used for non-Hodgkin's lymphoma (NHL): CVAD (cyclophosphamide, vincristine, doxorubicin, and dexamethasone), $\mathrm{CHOP}$ (cyclophosphamide, vincristine, doxorubicin, and prednisone) and ABV (doxorubicin, bleomycin, and vincristine) [16]. These regimens can be used alone or in combination with rituximab [17].

In patients with MCD relapse, retreatment with rituximab has proven effective [18]. Furthermore, patients who received Rituximab in addition to chemotherapy had a smaller risk of progressing to NHL and a higher 3-year overall survival rate (93 vs. 74\%) [19].

In patients with hematological complications like thrombocytopenia and anemia, splenectomy may be an additional therapeutic measure, although resolution of symptoms is usually not long lasting [20].

The available data on therapy and management of MCD relapses are limited and there is a lack of randomized trials in HIV- and HHV-8-positive patients. In conclusion, we provide our own experience with HIV-associated MCD with a cohort of patients diagnosed between 2008 and 2018.

\section{Methods}

We present a retrospective, descriptive study on a cohort of patients with MCD diagnosed and admitted to the infectious diseases or intensive care unit (ICU) in the University Hospital Düsseldorf between 2008 and 2018. We included all patients with previous or new HIV diagnosis and clinical signs resembling MCD in whom histological evidence of MCD was obtained or in whom HHV-8 DNA was detected in whole blood including peripheral blood mononuclear cells (PBMCs). The study was carried out in accordance with the code of ethics of the World Medical Association (Declaration of Helsinki) and was approved by the ethics committee of the University Hospital Düsseldorf.

\section{HHV-8 DNA quantification}

For HHV-8 quantification, DNA of $200 \mu \mathrm{l}$ whole blood was isolated automatically using the "EZ1 ${ }^{\circledR}$ DNA Blood Kit" with the EZ1 Advanced System (Qiagen). qPCR was performed by amplification of a fragment of the HHV-8 ORF26 gene region using the TaqMan Universal Master Mix (Applied Biosystems). The qPCR reaction was performed on an ABI 7500 Real-Time PCR system. HHV-8 DNA was normalized to the DNA amount of the patients' sample by quantification of the housekeeping gene beta-actin. Normalization to the cellular DNA was converted with the conversion factor 333 copies for $1 \mathrm{ng}$ DNA. qPCR results were

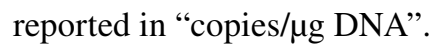

\section{HIV detection limit}

The lower limit of detection (LLD) for the HIV-1 RNA was 40 copies/ml (Abbott m2000 RealTime) until the end of 2014 when switching to a LLD of 20 copies/ml (COBAS AmpliPrep/TaqMan).

\section{Statistical analysis}

Simple frequencies, description and survival analysis were performed using SPSS Version 25.0.

\section{Results}

In total, nine male patients with a median age at diagnosis of 44 years (28-71 years) and a median time from HIV diagnosis of two years ( $0-19$ years) were included. Diagnosis was based on histopathological confirmation of HHV-8-positive MCD through a surgical lymph node extirpation in eight patients. In one patient (Patient 4), the diagnosis was confirmed based on clinical presentation and highly elevated HHV-8 viremia without histopathological proof [21]. The patients' characteristics as well as baseline parameters (at diagnosis of MCD), first-line therapies and outcomes are presented in Table 1.

Apart from common symptoms such as fever and weight loss, most patients presented at diagnosis with progressive generalized lymphadenopathy (8/9) and splenomegaly 


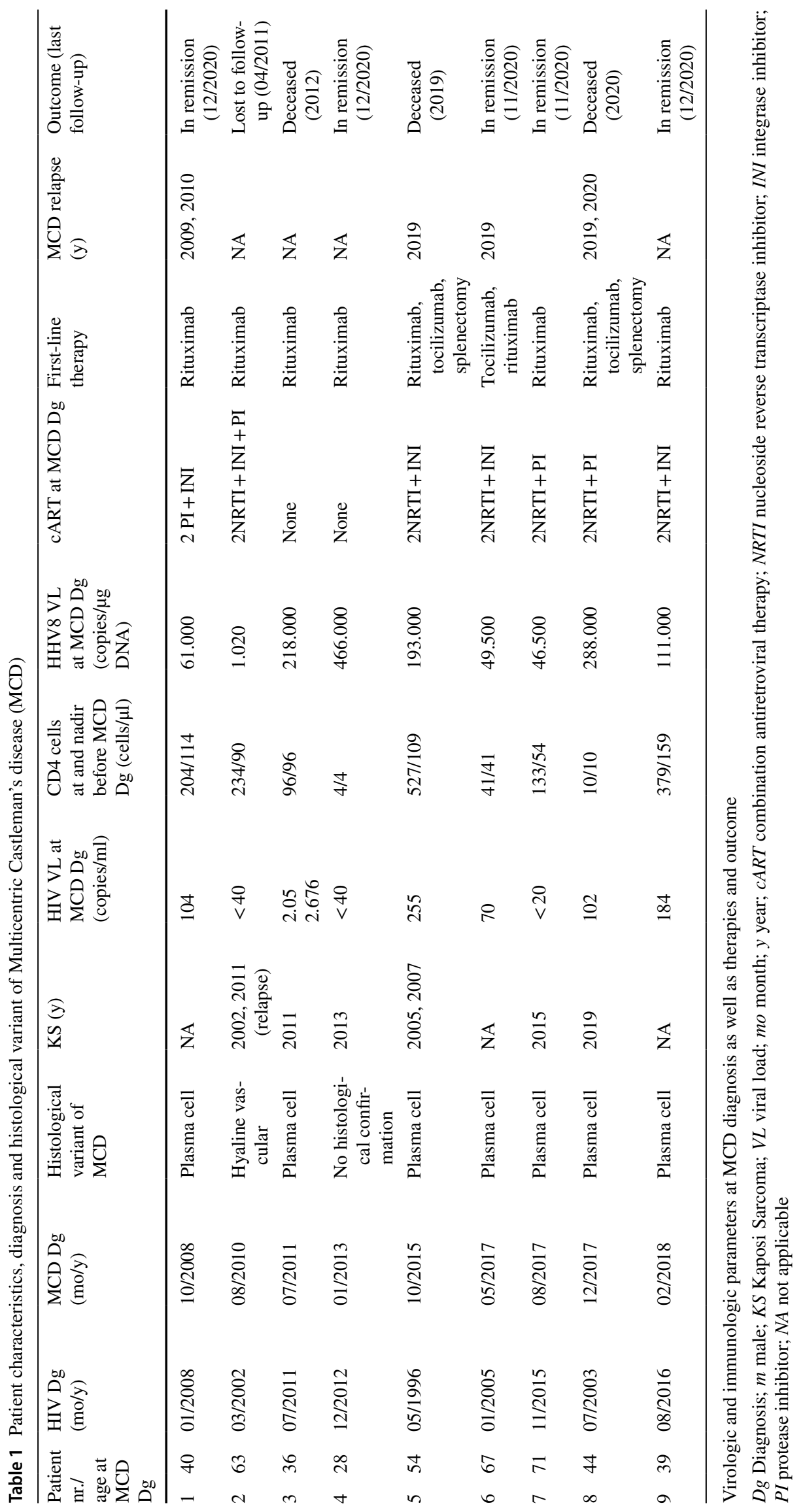


(9/9). Out of the seven patients who underwent bone marrow biopsy for diagnostic purposes, two had bone marrow involvement. Two patients presented with pulmonary involvement, one of whom had evidence of malignant cells in the pleural effusion, categorized as HHV-8-associated plasmablastic proliferation with features of a plasmacytoma. One patient developed polyneuropathy and endocrinopathies and POEMS syndrome was suspected, but the mandatory criteria of plasma cell dyscrasia and skin lesions were not present; therefore, the patient was classified as having a CD variant of POEMS syndrome.

Retrospectively, $78 \%$ of our patients (7/9) had a history of KS, which had been diagnosed before MCD in four (57\%) patients, coincidentally in two (29\%) and after MCD diagnosis in one (14\%) patient. Out of the four patients with prior KS diagnosis, three (75\%) had Kaposi's recurrence after treatment with a liposome-encapsulated anthracycline and before MCD diagnosis. Both patients with coincidental Kaposi's and MCD diagnosis (Patients 3 and 4) had bronchopulmonary manifestations of KS. Six patients $(86 \%)$ with KS had muco-cutaneous manifestations and three (43\%) had gastro-intestinal involvement. Four patients (57\%) received chemotherapy with liposome-encapsulated doxorubicin. Additionally, three patients (43\%) received topical radiotherapy of skin lesions, one of the latter patients (14\%) receiving additional chemotherapy with paclitaxel, which resulted in stable disease.

The median CD4 cell count at MCD diagnosis was 133/ $\mu \mathrm{l}$ (4-527) and median HHV-8 viral load (VL) was 111,000 copies/ $\mu$ g DNA (1020-466,000). Three patients had controlled HIV-1 infection (HIV-1-VL $<40$ copies/ml) at MCD diagnosis, one patient (patient 5) showed a blip of 255 cop$\mathrm{ies} / \mathrm{ml}$ at MCD diagnosis following previously suppressed HIV-1 viremia. The evolution of the CD4 cell count and HHV-8 DNA viral load is presented in Fig. 1. Interestingly, although some patients had suppressed HI-VL and four patients had CD4 cell counts $>200 / \mu \mathrm{l}$ (one patient $>500 / \mu \mathrm{l}$ ), all patients had CD4 cell counts below 200/ $\mu$ in their history (median nadir: 90/ $\mu \mathrm{l}$ (4-194)) before MCD diagnosis.

All patients were treated with rituximab, with resolution of symptoms and regression of lymphadenopathy and splenomegaly in six of them. Patients 4 and 7 received valganciclovir additionally.

Three patients received tocilizumab (patient 5,6 and 8), two of them after insufficient response to rituximab therapy and one as initial therapy with subsequent rituximab therapy (Patient 6). Patient 1 and 7, who received dual rituximab and tocilizumab therapy underwent cytoreductive surgery with splenectomy because of therapy refractory pancytopenia and in an attempt to reduce the HHV-8 reservoir [22]. Histological evidence of splenic involvement MCD was obtained in both patients. Human IL-6 levels were documented for patient 7 only, but were within normal range $(66-230 \mathrm{pg} /$
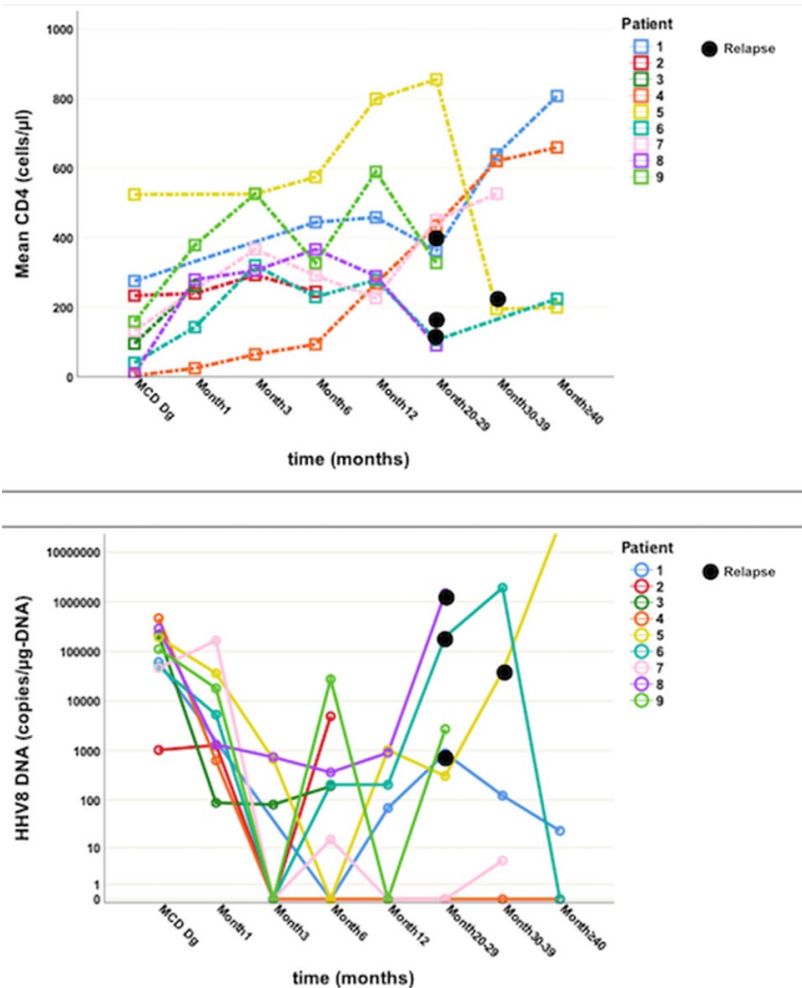

Fig. 1 Evolution of CD4 cell count (above) and HHV-8 DNA viral load in whole blood (below) after Multicentric Castleman's Disease (MCD) diagnosis. The HHV-8 DNA viral load is displayed using a logarithmic scale. The time axis is represented using a non-linear scale. Relapses are marked with black dots. Dg Diagnosis

$\mathrm{ml}$ ) and no significant change under tocilizumab therapy was observed.

Four patients had documented recurrences (Patients 1, 5, 6 and 8 ). Two of these patients ( 1 and 6 ) were treated again with 4 cycles of rituximab, with resolution of the disease. Patient 5 initially received treatment with tocilizumab, but he developed severe thrombocytopenia with platelet levels below $10,000 / \mu \mathrm{l}$ after the initial administration, which required repeated platelets transfusion. A switch to therapy with rituximab and vincristine was unsuccessful as the patient developed ARDS and metabolic acidosis and was transferred to the ICU. Concomitantly, the HHV-8 viremia exponentially increased up to a maximum of $37,849,102$ copies/ $\mu$ g DNA. A cytokine storm as complication of MCD or recurrence of a previously treated Listeria sepsis was suspected. In an effort to reduce cytokine levels and achieve sepsis and disease control, extracorporeal cytokine adsorber therapy with Cytosorb ${ }^{\circledR}$ and concurrent administration of siltuximab and the tyrosine kinase inhibitor ruxolitinib were started as salvage therapy. Under this intensified treatment, the patient's clinical condition initially improved significantly, accompanied by a decrease in HHV-8 viremia from $37,849,102$ to 3260 copies/ $\mu$ D DNA and a decrease in CRP 
from 17.0 to $0.7 \mathrm{mg} / \mathrm{dl}$. Unfortunately, the patient later died due to a sudden massive intracerebral hemorrhage in the setting of persistent marked thrombocytopenia.

Patient 8 initially received rituximab, after which he developed severe cytokine release syndrome and had to be admitted to the ICU. Under additional therapy with corticosteroids, Rituximab was continued but showed no marked improvement. Therefore - as performed with patient 5-salvage therapy with Cytosorb ${ }^{\circledR}$, ruxolitinib and tocilizumab was initiated. However, the patient failed to show any improvement and died.

With primary effusion lymphoma (PEL), one patient developed another HHV-8-associated malignancy, while another patient developed a diffuse large B-cell lymphoma (DLBCL).

Patient 8 presented with pleural and pericardial effusion and ascites and increasing HHV-8 viremia almost two years after MCD diagnosis. PEL was incidentally diagnosed with the MCD recurrence and treatment with cyclophosphamide, doxorubicin, prednisolone, and bortezomib (VcR-CAP protocol) was initiated. After initial improvement, the patient had to be readmitted due to complications of both lymphoma and MCD and later died. Patient 1 was diagnosed with DLBCL almost five years after MCD diagnosis and treated with 6 cycles of R-CHOP; the patient is now in remission.

Durable clinical remission after five years was documented in two patients (Patients 1 and 4). Patient 2 was lost to follow-up. Three patients, patient 3,5 and 8 died due to complications related to HIV and MCD. In conclusion, the survival rate in this study was $87.5 \%$ after 1 year (8/9), $71.4 \%$ after 3 years (5/7) and the relapse rate was $44 \%$ (4/9), occurring at a median of 30 months (range 20-36 months) after initial diagnosis and therapy of MCD.

\section{Discussion}

While $\mathrm{CD}$ is a rare diagnosis in the general population, MCD is an important differential diagnosis to consider in HIV-infected patients, even in the era of effective cART. All patients in our cohort are male, which reflects the epidemiology of HIV and especially HHV-8 in Germany.

Seven patients (78\%) presented with a plasma cell type MCD, out of which two had a KS-associated plasmablastic MCD. Seven out of nine patients had a previous, concurrent or later diagnosis of KS, findings that are similar to those reported in the literature $[5,6]$.

The median CD4 cell count at MCD diagnosis was 133 cells/ $\mu \mathrm{l}$, with a range between 4 and $527 \mathrm{n} / \mu \mathrm{l}$, which is slightly lower than values observed in other studies [23, 24]. In all nine patients however, a CD4 nadir $<200 / \mu$ was documented (median: 90 (range: 4-194)/ $\mu$ l) before MCD diagnosis, which is in contrast to previous studies, that suggested a higher risk of MCD in patients with a CD4 nadir > 200/ $\mu \mathrm{l}$ [25]. All patients were diagnosed in the cART era and $78 \%$ of them already received HIV therapy at MCD diagnosis. Out of these, 57\% (4/7) had a suppressed HIV-1 viral load, similar to values reported by Bower [23] and Loi et al. [26]. It has been shown that there is no relation between the use of cART or the current CD4 cell count and the incidence of MCD or MCD relapse in the HIV population $[26,27]$. One review of 72 HIV patients with MCD found that more than half of the patients diagnosed with MCD in the cART era had already received cART at the time of MCD diagnosis [6]. Although the reason for the apparent lack of a prophylactic effect of ART remains unknown, our data are consistent with previous publications. Eight out of nine patients showed high levels of HHV-8 DNA at MCD diagnosis (Fig. 1) and also during recurrence (if recurrence occurred). HHV-8 viremia at low levels (up to 2700 copies/ $\mu \mathrm{g}$ DNA) after treatment did not correlate with clinical signs of active MCD, confirming previously published data [7, 28]. Interestingly, the only patient with low HHV-8 DNA at time of diagnosis of MCD showed a hyaline vascular histological pattern, which is usually found in HIV-negative persons and, therefore, may represent a different pathophysiology of MCD. This patient was the only one to develop Kaposi's relapse despite suppressed HIV viral load, which is also consistent with an HHV-8 viremia of 4,910 copies/ $\mu \mathrm{g}$ DNA. While detectable HHV-8 viremia is almost always present at MCD diagnosis ( $\geq 95 \%)$ and its absence can be considered an exclusion criterion for HIV-associated MCD, HHV-8 viremia has shown poor correlation for other HIVassociated malignancies, which are often associated with other viruses like HPV and EBV [4].

Four patients with manifestations of KS before or concurrent to MCD diagnosis were treated with 6-8 cycles of pegylated liposomal doxorubicin, according to current guidelines [29]. Three patients were treated with radiotherapy for local disease, either alone or in combination with chemotherapy. Studies have shown that in patients with HHV-8-associated MCD, the concurrent use of rituximab and liposomal doxorubicin was noninferior to rituximab monotherapy and patients showed fewer KS exacerbations [30]. In our study, one out of two patients with MCD and concurrent KS received a combination therapy with doxorubicin and rituximab and had a durable clinical remission at five years. The other patient with HHV-8-associated MCD and KS received rituximab monotherapy but died due to reasons unrelated to KS exacerbation. KS exacerbation was observed in a different patient who received rituximab monotherapy and had up to that point not been treated for KS.

Following previous publications reporting successful outcomes [12, 13], all nine patients in our study were treated with immunotherapy, with eight of them receiving rituximab as first-line therapy. Rituximab was effective as 
initial agent in $63 \%$ of patients (5/8), two patients requiring additional therapy with tocilizumab. Relapses were seen one-three years after initial therapy for MCD in four patients, three of whom had initially received rituximab and tocilizumab therapy. Hence, the relapse rate shown in our patients is higher than described in previous studies: Bower et al. report a two-year survival rate of $94 \%$ and relapses in $22 \%$ with a median time to relapse of 2 years in a cohort of HIV-positive MCD treated with rituximab monotherapy [23]. Pria et al. report a 5-year overall survival of $92 \%$ and a 5-year relapse-free survival of $82 \%$ [18]. In both studies, patients who relapsed were again treated with rituximab-based therapy. In our study, MCD relapse therapy was also based on immunotherapy, with all four patients receiving rituximab. While two patients achieved complete remission following rituximab therapy, additional-ultimately unsuccessful-therapy was required in the other two patients.

In one of the cases, salvage therapy including ruxolitinib led to initial clinical improvement. The JAK inhibitor ruxolitinib has been used successfully in the treatment of hemophagocytic lymphohistiocytosis (HLH) [31], another hyperinflammatory syndrome, which shows some similarities in downstream cytokine activation with MCD, including the mTOR pathway. A recent study showed an increased mTOR activation in lymph node biopsies and PBMCs of patients with idiopathic MCD, which was reversible by JAK inhibition with ruxolitinib [32]. These data, as well as our observations, raise the possibility of a new therapeutic approach in rituximab refractory MCD cases. However, extensive studies are necessary in order to elucidate which role JAK inhibitors might finally play in the treatment of MCD. Additionally, it has to be mentioned that this approach failed to interrupt the cytokine storm in another patient and that in both patients, ruxolitinib was part of a combined salvage therapy, which prohibits attribution of the observed effect to a single agent.

Since tocilizumab and siltuximab inhibit human IL-6 (hIL-6) receptor binding only, symptom amelioration in a cytokine storm may be of therapeutic benefit. Viral IL6 (vIL-6) has been shown to bind directly to gp130 and is, therefore, independent of hIL-6 and its receptor, able to activate the subsequent signal-transduction cascade [33].

This is consistent with the observation that patients treated with tocilizumab during the first MCD episode required consolidation with rituximab. In a previous study, Nagao described two patients in whom tocilizumab showed initial clinical improvement but became ineffective as patients relapsed during continued treatment [11]. A different approach might be administration of rituximab simultaneously or closely followed by tocilizumab to achieve a faster response to therapy in severe cases of MCD.
Additive therapy, whether surgical (splenectomy) or conservative (antiviral therapy), was used in four patients, with the intention of controlling MCD-induced pancytopenia and HHV-8 viremia and because virus-activated cytotoxic therapy had proven useful in patients with mild HHV-8-associated MCD by reducing oral shedding of HHV-8 [34].

Two patients developed malignancies after initial diagnosis and therapy of MCD: HHV-8-associated primary effusion lymphoma and DLBCL, respectively. A study by Oksenhendler et al. showed a 15 -fold higher incidence of NHL in HIV patients with MCD than in the general HIV population [35]. While a reduced incidence of HHV-8-associated lymphomas in patients with previous diagnosis of MCD could be shown following the introduction of rituximab into MCD therapy, the rates remain still higher than in the overall population of people living with HIV $[18,19]$.

In conclusion, our study represents a single center's experience with a cohort of MCD in HIV patients and is one of the few studies in Germany that has been published in the past 10 years. To our knowledge, this is the first study reporting the use of JAK inhibitors in HIV-and HHV-8-associated MCD.

Treatment in our patients relied mostly on immunotherapy and in a couple of cases additive therapy, thus further underlining the effectiveness of rituximab as firstline therapy and retreatment during relapses. The role of tocilizumab as first-line therapy or in combination with rituximab needs to be further investigated. Although our experiences suggest the effectiveness of other treatment options like splenectomy or different immunotherapeutic approaches, including ruxolitinib, these findings require confirmation.

Our study is limited by the fact that we did not determine hIL-6 and vIL-6 levels in all patients receiving tocilizumab, thus providing a hindsight into the mechanism of action during initial therapy and relapses in HIV patients.

Future studies on larger number of patients are needed to improve the current guidelines of management and treatment in HIV- and HHV-8-associated MCD and will require cross-hospital or country collaborations given the overall low incidence of HIV/HHV-8-associated MCD.

Author contribution S. G., H. M. O., N. L., T.L. and B. J. wrote the manuscript. S. G. and H. M. O. collected the data and performed the statistical analysis. N. L. and J. T. supervised the virologic assays. All authors approved the final manuscript.

Funding The authors received no further funding for this work.

Availability of data and material The data that support the findings of this study are available from the corresponding author, S.G., upon request. 


\section{Declarations}

Conflict of interest The authors declare no conflicts of interest.

\section{References}

1. Castleman B, Towne VW. Case records of the Massachusetts General Hospital: case no. 40231. N Engl J Med. 1954;250:1001-5.

2. Carbone A, De Paoli P, Gloghini A, Vaccher E. KSHV-associated multicentric Castleman disease: a tangle of different entities requiring multitarget treatment strategies. Int $\mathrm{J}$ Cancer. 2015;137:251-61.

3. His ED, Lorsbach RB, Fend F, Dogan A. Plasmablastic lymphoma and related disorders. Am J Clin Pathol. 2011;136:183-94.

4. Haq I-U, Pria AD, Papanastasopoulos P, Stegmann K, Bradshaw D, Nelson M, Bower M. The clinical application of plasma Kaposi sarcoma herpesvirus viral load as a tumour biomarker: results from 704 patients. HIV Med. 2016;17:56-61.

5. Soulier J, Grollet L, Oksenhendler E, et al. Kaposi's sarcomaassociated herpesvirus-like DNA sequences in multicentric Castleman's disease. Blood. 1995;86:1276-80.

6. Mylona EE, Baraboutis IG, Lekakis LJ, et al. Multicentric Castleman's disease in HIV infection: a systematic review of the literature. AIDS. 2008; 10:25-35.

7. Sayer R, Paul J, Tuke PW, Hargreaves S, Noursadeghi M, Tedder RS, Grant P, Edwards SG, Miller RF. Can plasma HHV8 viral load be used to differentiate multicentric Castleman disease from Kaposi sarcoma? Int J STD AIDS. 2011;22:585-9.

8. Nishimoto N, Kanakura Y, Aozasa K, Johkoh T, Nakamura M, Nakano S, Nakano N, Ikeda Y, Sasaki T, Nishioka K, Hara M, Taguchi H, Kimura Y, Kato Y, Asaoku H, Kumagai S, Kodama F, Nakahara H, Hagihara K, Yoshizaki K, Kishimoto T. Humanized anti-interleukin- 6 receptor antibody treatment of multicentric Castleman disease. Blood. 2005;106:2627-32.

9. Koff JL, Lonial S. Emerging treatments in Castleman disease - a critical appraisal of siltuximab. Biologics. 2016;10:9-15.

10. Kurzrock R, Voorhees PM, Casper C, et al. A phase I, open-label study of siltuximab, an anti-IL-6 monoclonal antibody, in patients with B-cell non-Hodgkin lymphoma, multiple myeloma, or Castleman disease. Clin Cancer Res. 2013;19:3659-70.

11. Nagao A, Nakazawa S, Hanabusa H. Short-term efficacy of the IL6 receptor antibody tocilizumab in patients with HIV-associated multicentric Castleman disease: report of two cases. J Hematol Oncol. 2014;7:10.

12. Bower M, Powles T, Williams S, et al. Brief communication: rituximab in HIV-associated multicentric Castleman disease. Ann Intern Med. 2007;147:836-9.

13. Hoffmann C, Schmid H, Müller M, Teutsch C, van Lunzen J, Esser S, Wolf T, Wyen C, Sabranski M, Horst HA, Reuter S, Vogel M, Jäger H, Bogner J, Arasteh K. Improved outcome with rituximab in patients with HIV-associated multicentric Castleman disease. Blood. 2011;118:3499-503.

14. Neuville S, Agbalika F, Rabian C, Briere J, Molina JM. Failure of rituximab in human immunodeficiency virus-associated multicentric Castleman disease. Am J Hematol. 2005;79:337-9.

15. Buchler T, Dubash S, Lee V, et al. Rituximab failure in fulminant multicentric HIV/human herpesvirus 8-associated Castleman's disease with multiorgan failure: report of two cases. AIDS. 2008;22:1685-7.

16. Oksenhendler E, Duarte M, Soulier J, et al. Multicentric Castleman's disease in HIV infection: a clinical and pathological study of 20 patients. AIDS. 1996;10:61-7.
17. Bower M. How I treat HIV-associated multicentric Castleman disease. Blood. 2010;116:4415-21.

18. Pria AD, Pinato D, Roe J, Naresh K, Nelson M, Bower M. Relapse of HHV8-positive multicentric Castleman disease following rituximab-based therapy in HIV-positive patients. Blood. 2017;129:2143-7.

19. Gérard L, Michot JM, Burcheri S, Fieschi C, Longuet P, Delcey V, Meignin V, Agbalika F, Chevret S, Oksenhendler E, Galicier L. Rituximab decreases the risk of lymphoma in patients with HIV-associated multicentric Castleman disease. Blood. 2012;119:2228-33.

20. Waterston A, Bower M. Fifty years of multicentric Castleman's disease. Acta Oncol. 2004;43:698-704

21. Bower M, Pria AD, Coyle C, Nelson M, Naresh K. Diagnostic criteria schemes for multicentric Castleman disease in 75 cases. J Acquir Immune Defic Syndr. 2014;65:e80-2.

22. Hoffmann C, Rockstroh J.K. (2018) HIV 2018/2019. Medizin Fokus Verlag, pp 412-20

23. Bower M, Newsom-Davis T, Naresh K, et al. Clinical features and outcome in HIV-associated multicentric Castleman's disease. J Clin Oncol. 2011;29:2481-6.

24. Mahroug ER, Sher-Locketz C, Desmirean MS, Abayomi EA, Tomuleasa C, Grewal R. Castleman's disease in the HIV-endemic setting. Cancer Manag Res. 2018;10:4553-63.

25. Powles T, Stebbing J, Bazeos A, et al. The role of immune suppression and HHV-8 in the increasing incidence of HIV-associated multicentric Castleman's disease. Ann Oncol. 2009;20:775-9.

26. Loi S, Goldstein D, Clezy K, Milliken ST, Hoy J, Chipman M. Castleman's disease and HIV infection in Australia. HIV Med. 2004;5:157-62.

27. Oksenhendler E, Carcelain G, Aoki Y, et al. High levels of human herpesvirus 8 viral load, human interleukin-6, interleukin-10, and $\mathrm{C}$ reactive protein correlate with exacerbation of multicentric Castleman disease in HIV-infected patients. Blood. 2000;96:2069-73.

28. Polizzotto MN, Uldrick TS, Wang V, Aleman K, Wyvill KM, Marshall V, Pittaluga S, O'Mahony D, Whitby D, Tosato G, Steinberg SM, Little RF, Yarchoan R. Human and viral interleukin-6 and other cytokines in Kaposi sarcoma herpesvirus-associated multicentric Castleman disease. Blood. 2013;122:4189-98.

29. Hoffmann C, Sabranski M, Esser S. HIV-associated Kaposi's sarcoma. Oncol Res Treat. 2017;40:94-8.

30. Uldrick TS, Polizzotto MN, Aleman K, et al. Rituximab plus liposomal doxorubicin in HIV-infected patients with KSHV-associated multicentric Castleman disease. Blood. 2014;124:3544-52.

31. Keenan C, Nichols KE, Albeituni S. Use of the JAK inhibitor ruxolitinib in the treatment of hemophagocytic lymphohistiocytosis. Front Immunol. 2021;12:614704.

32. Arenas DJ, Floess K, Kobrin D, Pai RL, Srkalovic MB, Tamakloe MA, Rasheed R, Ziglar J, Khor J, Parente SAT, Pierson SK, Martinez D, Wertheim GB, Kambayashi T, Baur J, Teachey DT, Fajgenbaum DC. Increased mTOR activation in idiopathic multicentric Castleman disease. Blood. 2020;135:1673-84.

33. Müllberg J, Geib T, Jostock T, Hoischen SH, Vollmer P, Voltz N, Heinz D, Galle PR, Klouche M, Rose-John S. IL-6 receptor independent stimulation of human gp130 by viral IL-6. J Immunol. 2000;164:4672-7.

34. Uldrick TS, Polizzotto MN, Aleman K, et al. High-dose zidovudine plus valganciclovir for Kaposi sarcoma herpesvirusassociated multicentric Castleman disease: a pilot study of virusactivated cytotoxic therapy. Blood. 2011;117:6977-86.

35. Oksenhendler E, Boulanger E, Galicier L, et al. High incidence of Kaposi sarcoma-associated herpesvirus-related non-Hodgkin lymphoma in patients with HIV infection and multicentric Castleman disease. Blood. 2002;99:2331-6. 